Supporting information

\title{
Spray Injection Direct Analysis in Real Time (DART) Ionization for Petroleum
}

\section{Analysis}

Limin Ren, Yehua Han ${ }^{*}$, Yahe Zhang, Yanfen Zhang, Xianghai Meng, Quan Shi*

State Key Laboratory of Heavy Oil Processing, China University of Petroleum, Beijing 102249, China

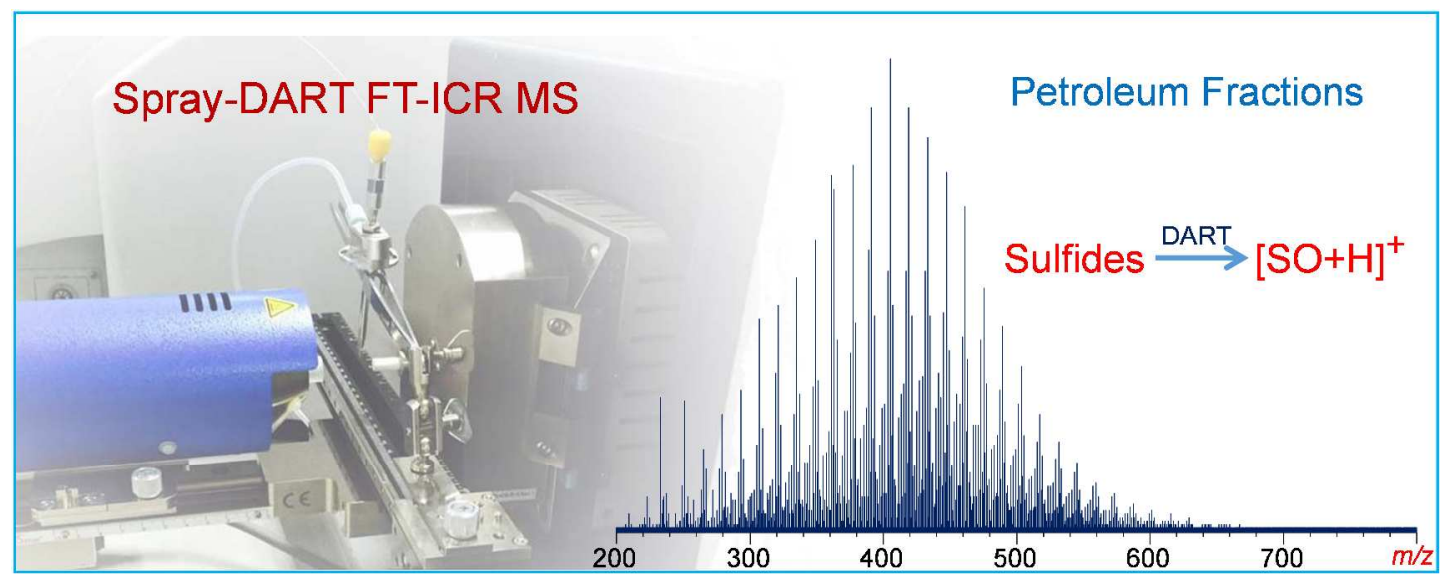



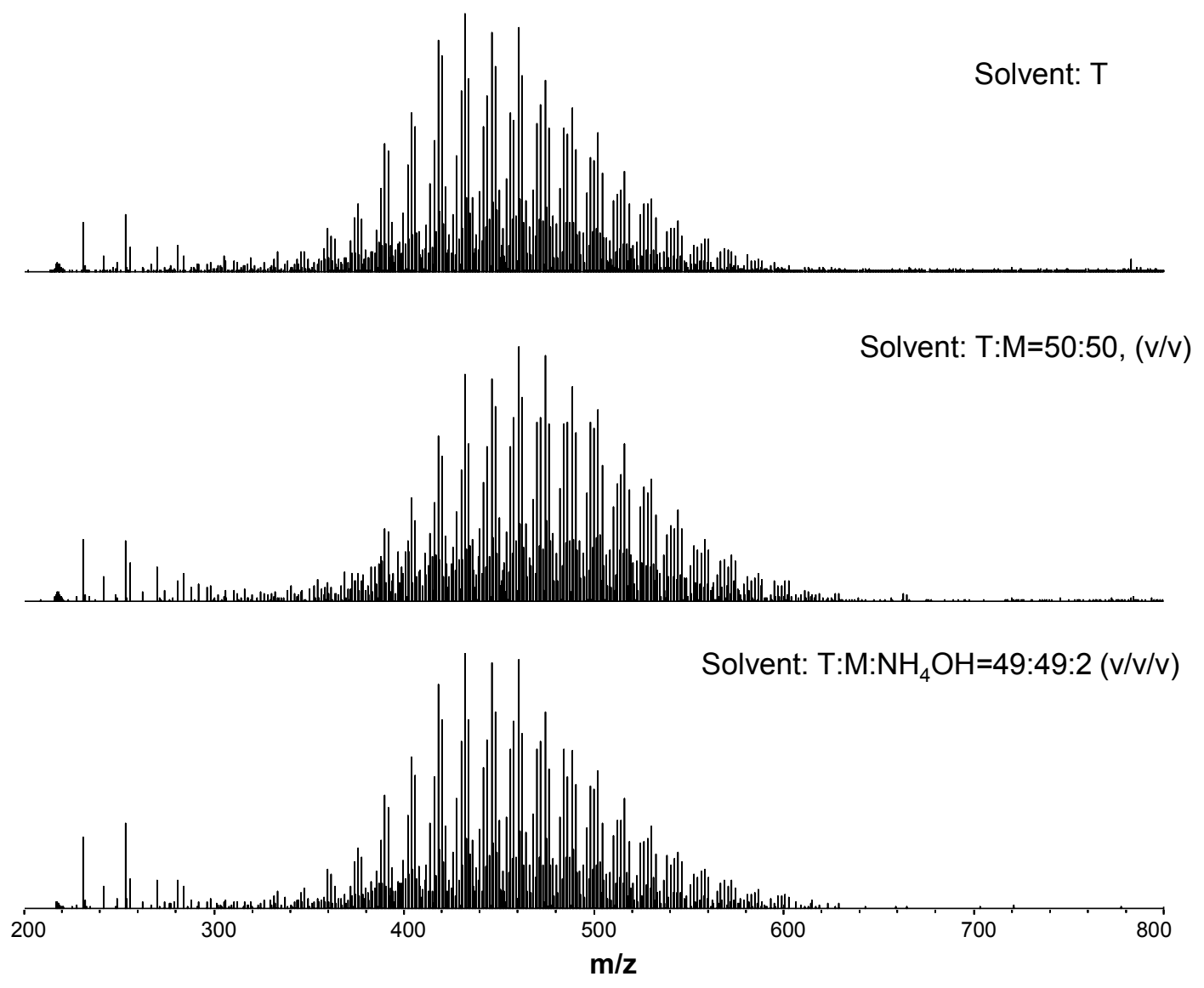

Figure S1. Negative (-) mode Spray-DART FT-ICR mass spectra of the crude oil \#1 obtained with toluene (top), toluene-methanol (50:50, v/v) (middle), and an ammonium hydroxide-modified solvent (bottom). 


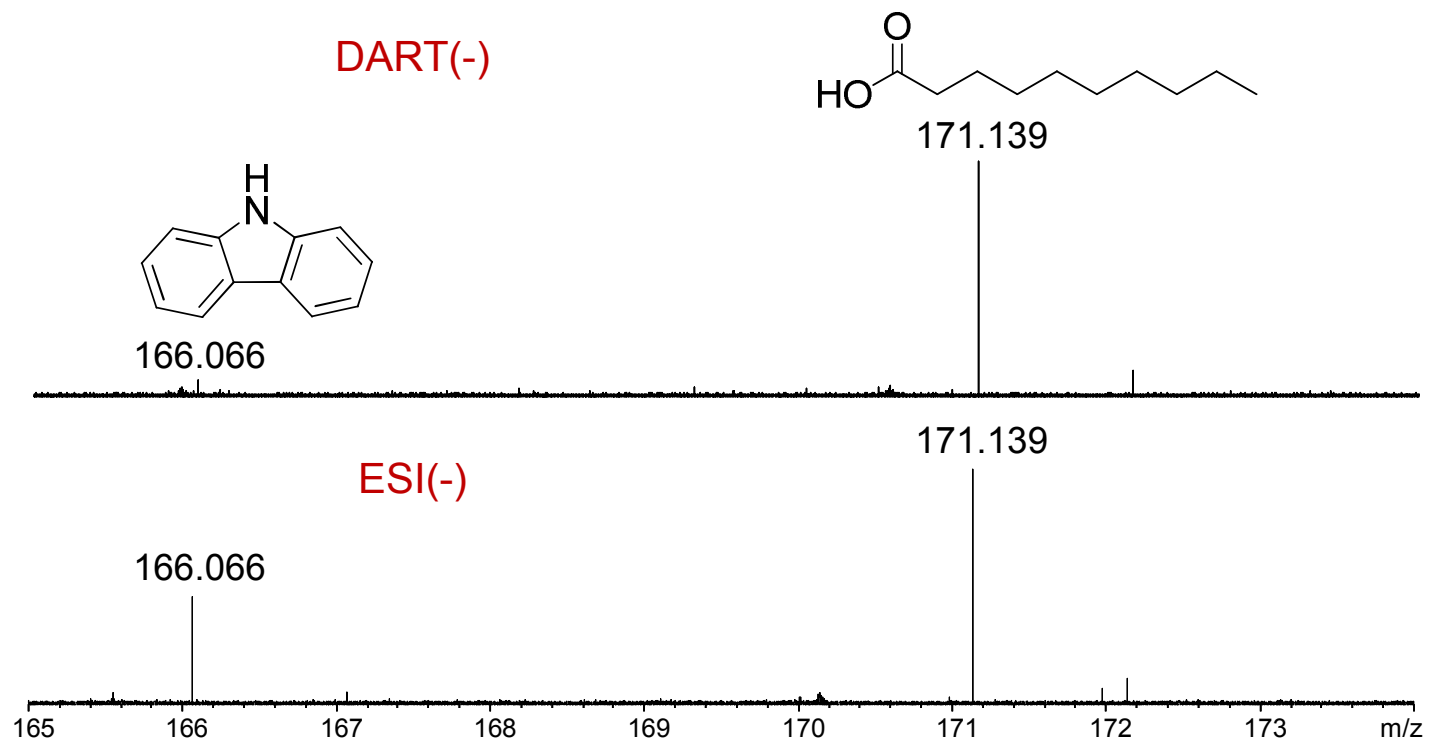

Figure S2. FT-ICR mass spectra of standard compounds (carbazole, decanoic acid) obtained by DART and ESI with the mole concentration ratio of 1:1.
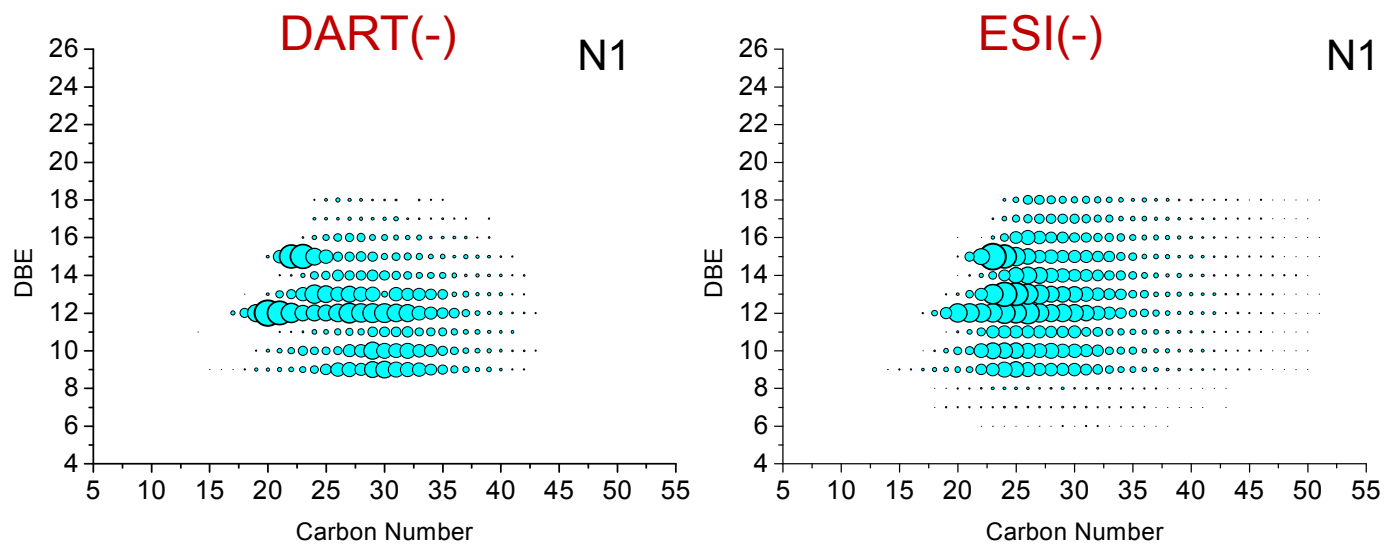

Figure S3. Plots of DBE versus carbon number for N1 class species assigned from DART (-) and ESI (-) mass spectra of crude oil \#1. 
(a)

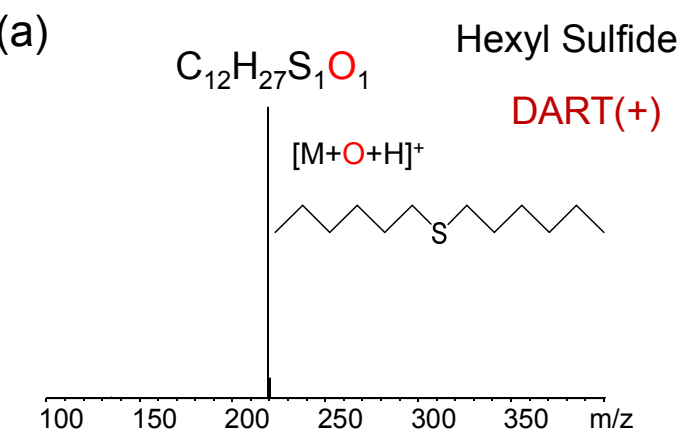

(c)

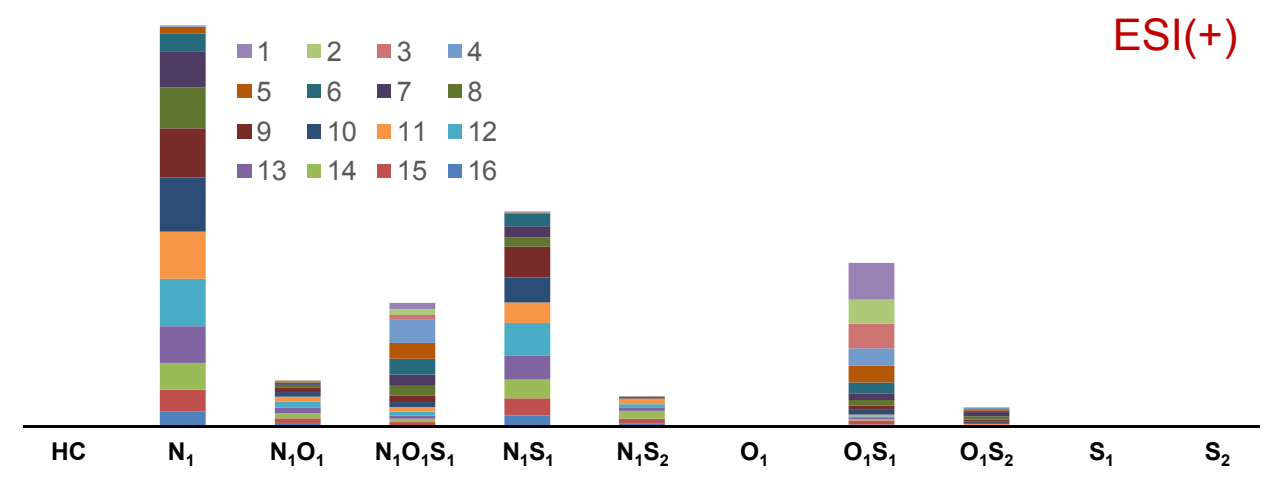

(b)

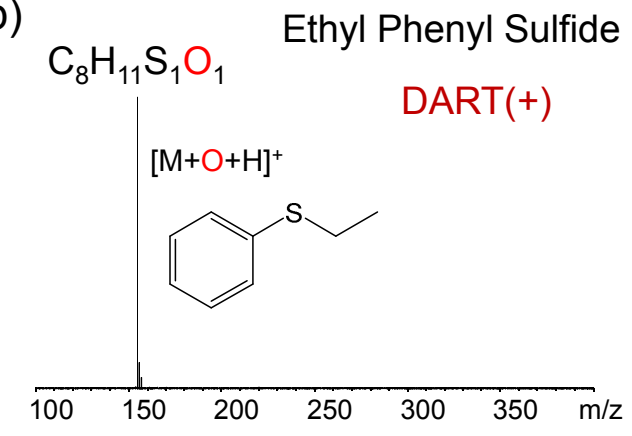

Figure S4. DART (+) FT-ICR mass spectra of model compounds with different structures:

(a) hexyl sulfide, (b) ethyl phenyl sulfide. The $[\mathrm{M}+\mathrm{O}+\mathrm{H}]^{+}$ions should be the oxidation products of the corresponding sulfides. (c) Class species detected by ESI (+) FT-ICR MS of the Kuwait crude oil. 


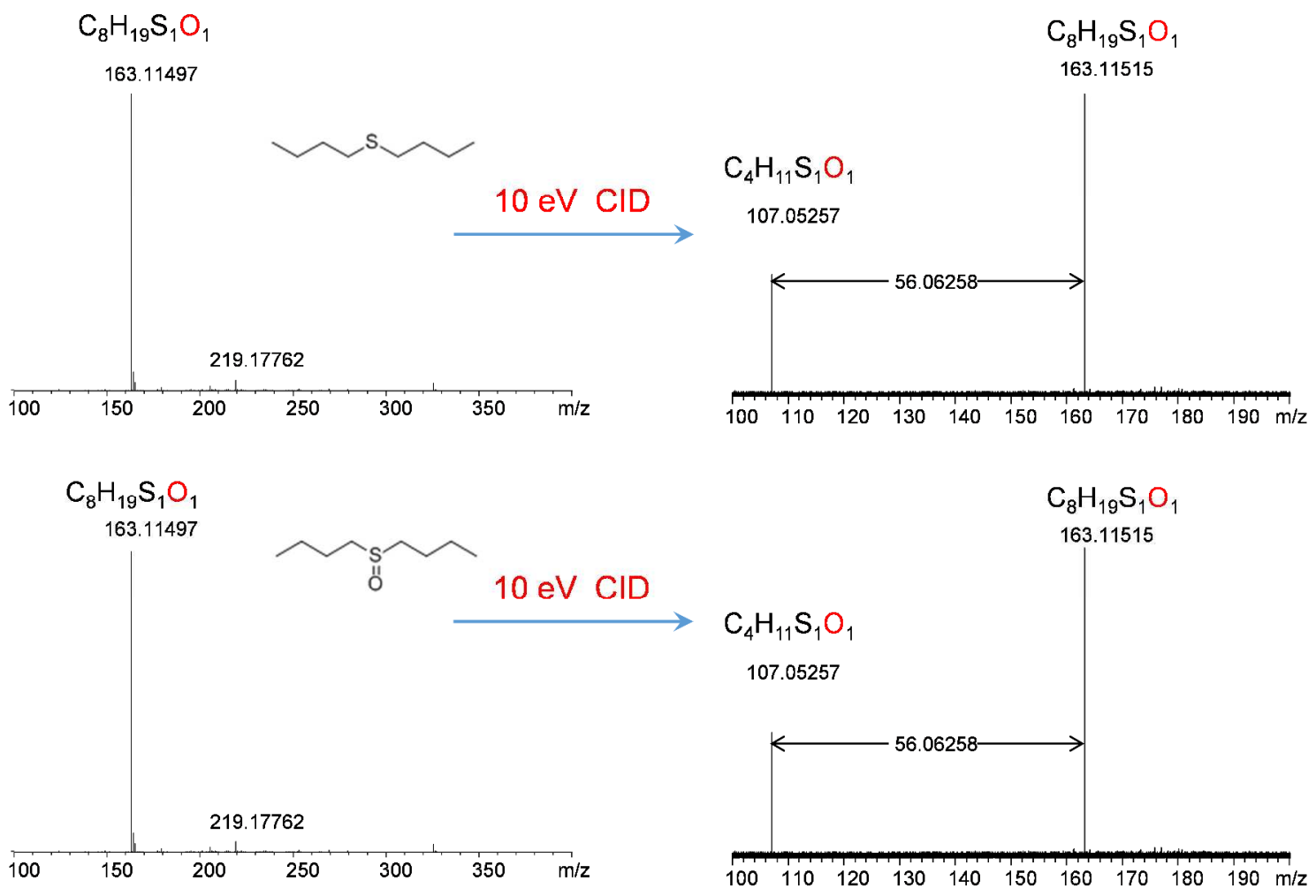

Figure S5. DART(+) mass spectra of butyl sulfide and butyl sulfoxide (left); CID mass spectra of DRAR(+) mass spectra of butyl sulfide and butyl sulfoxide (right). 

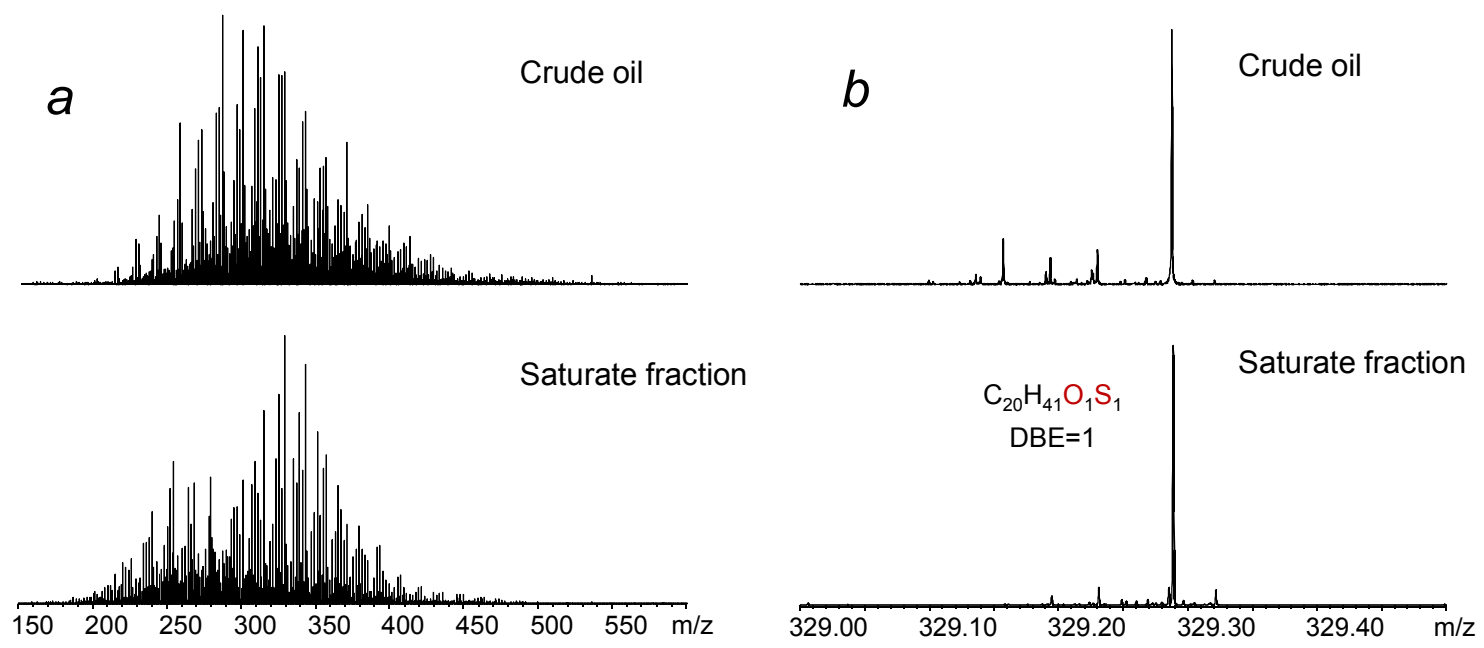

Figure S6. Broad bond (a) and expended (b) DART (+) FT-ICR mass spectra of the Kuwait crude oil and its saturate fraction. 

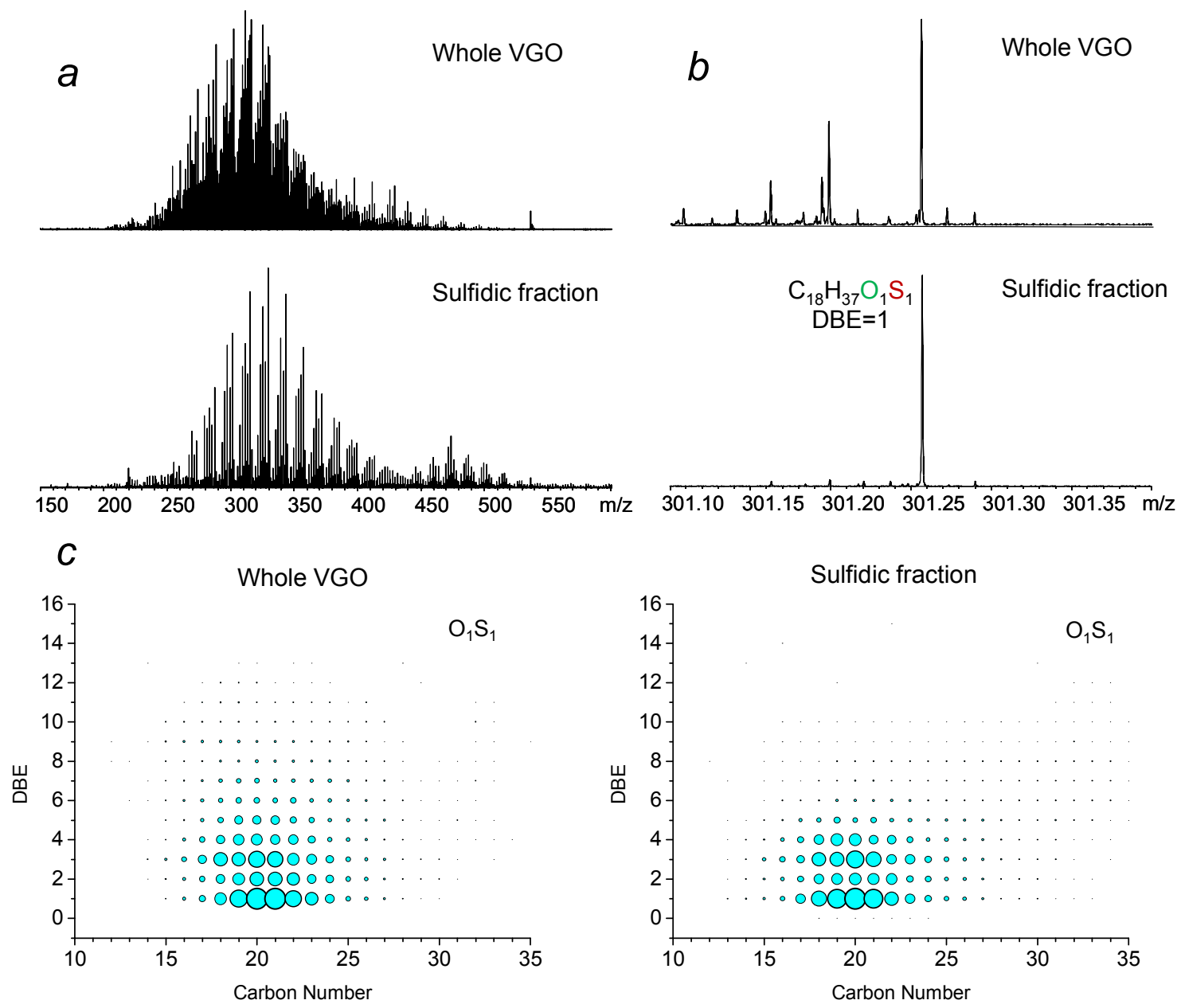

Figure S7. Broad bond (a) and expended (b) DART (+) FT-ICR mass spectra of the VGO and its sulfidic fraction. The fraction was obtained from the Kuwait crude oil with a distillation temperature range of $350-400{ }^{\circ} \mathrm{C}$. (c) Distribution of DBE versus carbon number of O1S1 class species assigned from the mass spectra. Sulfidic fraction was separated by methylation/demethylation method (Reference 53, Wang, M., Zhao, S., Chung, K. H., Xu, C., and Shi, Q., Approach for Selective Separation of Thiophenic and Sulfidic Sulfur Compounds from Petroleum by Methylation/Demethylation. Analytical Chemistry, 2015. 87(2): 1083-1088). 

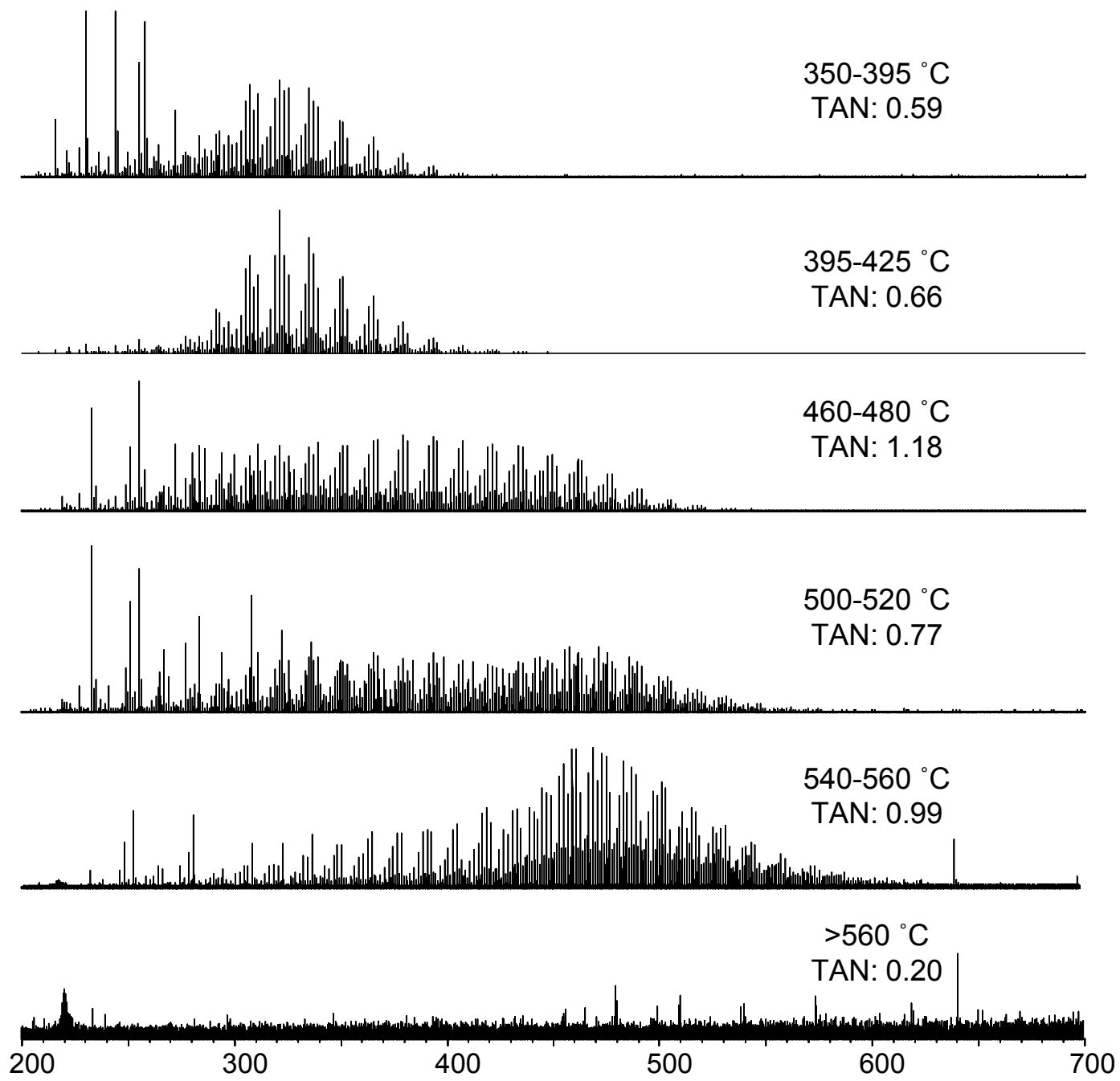

Figure S8. FT-ICR mass spectra of a set of VGO fractions and a residual obtained using DART in negative mode. From top to bottom are fractions with boiling range of $350-395{ }^{\circ} \mathrm{C}, \quad 395-425{ }^{\circ} \mathrm{C}, 460-480{ }^{\circ} \mathrm{C}, 500-520{ }^{\circ} \mathrm{C}, 540-560{ }^{\circ} \mathrm{C}$, and $>560{ }^{\circ} \mathrm{C}$, respectively. 